\title{
Evolution of the pairing pseudogap in the spectral function with interplane anisotropy
}

\author{
G. Preosti ${ }^{1}$, Y. M. Vilk ${ }^{2}$, and M. R. Norman ${ }^{1}$ \\ (1) Materials Sciences Division, Argonne National Laboratory, Argonne, IL 60439 \\ (2) 2100 Valencia Dr. Apt. 406, Northbrook, IL 60062
}

(August 9, 2018)

\begin{abstract}
We study the pairing pseudogap in the spectral function as a function of interplane coupling. The analytical expressions for the self-energy in the critical regime are obtained for any degree of anisotropy. The frequency dependence of $\Sigma(\omega)$ is found to be qualitatively different in two and three dimensions, and the crossover from two to three dimensional behavior is discussed. In particular, by considering the anisotropy of the Fermi velocity and gap along the Fermi surface, we can qualitatively explain recent photoemission experiments on high temperature superconductors concerning the temperature dependent Fermi arcs seen in the pseudogap phase.
\end{abstract}

\section{INTRODUCTION}

It is clearly shown by experimental data that underdoped cuprates exhibit a large suppression of low energy spectral weight at temperatures higher than the superconducting (SC) critical temperature, which constitutes the so called pseudogap phenomenon. Early evidence came from a number of probes, such as NMR, infrared reflectivity, and specific heat [1], while recent angle-resolved photoemission (ARPES) experiments have added qualitatively new information, and attracted considerable attention to this phenomenon [2] [3]. The picture that emerges from ARPES data is that of a highly anisotropic pseudogap, for $T_{c}<T<T^{*}$, which in magnitude and angular dependence resembles the superconducting gap below $T_{c}$ and smoothly evolves into it as the temperature is lowered. With increasing underdoping, the interplane anisotropy of these materials increases $\mathbb{\|}, T_{c}$ goes down, and 
the amplitude of the gap and the temperature $T^{*}$, at which the pseudogap opens, increases, thus widening the extent of the pseudogap phase. Moreover the temperature $T^{*}$, and the strength of the pseudogap feature, are strongly dependent on the region of the Fermi surface that is probed, being quite enhanced close to the $(\pi, 0)$ point and vanishing along the diagonal direction in the Brillouin zone.

These measurements have spawned a number of theoretical interpretations, and no consensus has yet being reached. Theoretical models span a wide range of possible mechanisms, including RVB-like pairing of chargeless spinons [5], antiferromagnetic correlations [6], and preformed pairs [0]. Here we will concentrate on the effects of dimensionality [8] and intraplane anisotropy on the properties of the pseudogap.

It is the purpose of this work to show under what general conditions an observable pseudogap can be expected as a precursor of superconductivity in the weak to intermediate coupling regime, and to demonstrate the crucial role of both in plane and interplane anisotropy in determining the properties of the pseudogap. We adopt a procedure for obtaining an analytical estimate of the electron self energy in the renormalized classical regime of the pairing fluctuations, which is similar to the one adopted by Vilk et. al. 9, 10. This procedure is justifiable up to intermediate coupling.

First we find that a very weak pseudogap exists in the spectral function $A(\omega, \mathbf{k})$ even in a perfectly isotropic three dimensional (3D) superconductor but it is of a very different nature from the one found in exactly two spatial dimensions, and even very close to the transition temperature it would be practically unobservable at present day resolution. By contrast, for highly anisotropic materials, the size of the pseudogap well above the SC critical temperature becomes almost the same as in the ordered state. Therefore, unless strong coupling is invoked, a high interplane anisotropy is required to make the pseudogap observable. The condition for having a strong quasi-2D pseudogap effect in $A(\omega, \mathbf{k})$ can be expressed as: $\Delta_{\mathbf{k}}(0) \gtrsim \omega_{X}$, where $\Delta_{\mathbf{k}}(0)$ is the k-dependent value of the zero temperature gap amplitude, and $\omega_{X}=\left(\xi_{z} / \xi_{x y}\right) v_{F}(\mathbf{k}) q_{B Z}^{(z)}$ is a dimensional crossover frequency which involves both the interplane anisotropy through the pairing correlation length anisotropy ratio $\xi_{z} / \xi_{x y}$, and the 
in plane anisotropy of the Fermi velocity $v_{F}(\mathbf{k})$ (here $q_{B Z}^{(z)}$ is the Brillouin zone boundary in the direction of the anisotropy axis). Second, we find that all the properties of the pseudogap, and in particular its temperature evolution, are strongly affected by the ratio $\Delta_{\mathbf{k}}(0) / v_{F}(\mathbf{k})$, which depends on the particular point probed on the Fermi surface. We find that this dependence on the in plane anisotropy naturally accounts for the ARPES observation of Fermi arcs in the pseudogap regime, and also the apparently different line shape and temperature dependence of the pseudogap at different points on the Fermi surface. In particular, we explain the experimental observation that the pseudogap seems to fill up close to the $(\pi, 0)$ point, and to close in for points close to the zone diagonal.

\section{FORMALISM}

We evaluate the effects of pairing fluctuations on the electronic self-energy of anisotropic superconductors in the one loop approximation. The expression for the self energy has the form:

$$
\Sigma(\omega, \mathbf{k})=g_{\mathbf{k}}^{2} \int \frac{d^{3} q d \omega^{\prime}}{2 \pi \Omega} \frac{\chi^{\prime \prime}\left(\omega^{\prime}, \mathbf{q}\right)\left\{\operatorname{coth}\left(\frac{\omega^{\prime}}{2 T}\right)-\tanh \left(\frac{\widetilde{\varepsilon}(-\mathbf{k}+\mathbf{q})}{2 T}\right)\right\}}{\omega-\omega^{\prime}+\widetilde{\varepsilon}(-\mathbf{k}+\mathbf{q})+i 0^{+}}
$$

where $g_{\mathbf{k}}^{2}$ is the effective coupling constant [1] between electrons and pairing fluctuations, $\Omega$ the volume of the reciprocal lattice primitive cell, $\widetilde{\varepsilon}(\mathbf{k})$ the energy dispersion relative to the chemical potential, and $\chi(\omega, \mathbf{q})$ the pairing susceptibility. In this formula and what follows we have set the in plane lattice constant $a$ as the unit of length, the interplane lattice constant as $a_{z}=\pi / q_{B Z}^{(z)}$, and we also consider natural units: $\hbar=k_{B}=1$.

In the critical regime close to the $\mathrm{SC}$ instability, the pairing susceptibility $\chi$ is strongly peaked at $q=0, \omega=0$. It is this peak in the pairing susceptibility that gives rise to the pseudogap effect. For the purpose of this work, it will be sufficient to assume that for $T<T_{X}$, where $T_{X}$ is the crossover temperature to the critical regime, $\chi$ can be approximated in the neighborhood of $(\omega, \mathbf{q})=0$ by the following Lorentzian form:

$$
\chi(\omega, \mathbf{q})=\frac{\chi_{0}\left(0,0, T_{X}\right)}{\xi_{0}^{2}} \frac{1}{\xi^{-2}-\frac{i \omega}{D}+\left(q_{x}^{2}+q_{y}^{2}\right)+\gamma^{2} q_{z}^{2}}
$$


where $\chi_{0}\left(0,0, T_{X}\right)$ is the pairing susceptibility for non-interacting electrons at the crossover temperature, $\xi_{0}=\xi_{0 x y}$ is the in plane coherence length, and $D$ the microscopic diffusion constant. Two other fundamental quantities appear in this expression: the in plane correlation length $\xi=\xi_{x y}$ which diverges at the transition temperature, and the anisotropy parameter $\gamma=\xi_{0 z} / \xi_{0}$ which expresses the interplane anisotropy. In the pairing channel, the expansion for $\chi^{-1}(\omega, \mathbf{q})$ can also contain a real linearly dependent frequency term, $\omega / D_{1}$. However, it can be shown by direct evaluation of the bare susceptibility $\chi_{0}(\omega, \mathbf{q})=-\left[\frac{1}{\beta} \sum_{i k_{n}} \sum_{\mathbf{k}} G^{0}\left(i k_{n}, \mathbf{k}\right) G^{0}\left(i q_{n}-i k_{n}, \mathbf{q}-\mathbf{k}\right)\right]_{i q_{n \rightarrow \omega+i \delta}}$ that at the beginning of the critical regime $D=\left(\frac{-1}{\xi_{0}^{2} \chi_{0}} \operatorname{Im}\left[\frac{\partial \chi_{0}(q, 0)}{\partial \omega}\right]\right)^{-1} \sim T$, while $D_{1}=\left(\frac{1}{\xi_{0}^{2} \chi_{0}} \operatorname{Re}\left[\frac{\partial \chi_{0}(q, 0)}{\partial \omega}\right]\right)^{-1} \sim E_{F}$, and therefore the term $\omega / D_{1}$ should be negligible with respect to the $i \omega / D$ term in a degenerate electron system. Since ARPES data show a large Fermi surface [13] we neglect this additional term. The case in which the $\omega / D_{1}$ term is dominant has being considered in references [8, 12].

In mean field theory, the correlation length $\xi$ diverges as $1 / \sqrt{T-T_{c}}$. However, mean field theory violates the Mermin-Wagner theorem [14], and therefore cannot give a viable description of the effects of pairing fluctuations in low dimensional systems. In order to go beyond mean field theory we will utilize the fluctuation-dissipation theorem:

$$
\int \frac{d^{3} q}{\Omega} \int \frac{d \omega}{2 \pi} \chi^{\prime \prime}(\omega, \mathbf{q}) \operatorname{coth} \frac{\omega}{2 T}=\frac{1}{2}\left(\left\langle\widetilde{\Delta} \widetilde{\Delta}^{+}\right\rangle+\left\langle\widetilde{\Delta}^{+} \widetilde{\Delta}\right\rangle\right)
$$

where $\widetilde{\Delta}=\sum_{j} \eta_{i j} c_{i \uparrow} c_{j \downarrow}$ is the local pairing [15 operator and $\langle\ldots\rangle$ indicates thermodynamic averaging. In order to satisfy the Mermin-Wagner theorem, the right hand side of eq. (3) must remain finite (the mean field assumption leads to a divergence in 2D [9]). Moreover, the phase fluctuation scenario consists in having a state with a non-zero local superconducting pairing amplitude, but no off diagonal long range order due to thermal fluctuations in the phase of the order parameter. It is important to realize that the locally ordered part of the pairing amplitude cannot be simply identified with the full correlator in equation (3) because the latter quantity is large and nonzero even in the non-interacting system. In order to make the connection between the local pairing amplitude in the pseudogap 
regime and the integral of $\chi^{\prime \prime}(\omega, \mathbf{q})$, we first note that Monte Carlo simulations [16] show that the temperature dependence of the quantity $\frac{1}{2}\left(\left\langle\widetilde{\Delta} \widetilde{\Delta}^{+}\right\rangle+\left\langle\widetilde{\Delta}^{+} \widetilde{\Delta}\right\rangle\right)$ goes through a minimum at some crossover temperature $T_{X}$, and below $T_{X}$ it increases as if it were in the ordered state. Clearly this growth of the $\frac{1}{2}\left(\left\langle\widetilde{\Delta} \widetilde{\Delta}^{+}\right\rangle+\left\langle\widetilde{\Delta}^{+} \widetilde{\Delta}\right\rangle\right)$ correlator with decreasing temperature is due to the contribution of thermal critical fluctuations. We thus identify the local superconducting pairing amplitude with the almost singular contribution of the classical fluctuations to the correlator $\frac{1}{2}\left(\left\langle\widetilde{\Delta}^{+}\right\rangle+\left\langle\widetilde{\Delta}^{+} \widetilde{\Delta}\right\rangle\right)$ :

$$
\int \frac{d^{3} q}{\Omega} \int \frac{d \omega}{2 \pi} \chi^{\prime \prime}(\omega, \mathbf{q})\left[\operatorname{coth} \frac{\omega}{2 T}-\operatorname{sign}(\omega)\right]=\widetilde{\Delta}_{c l}^{2}(T) \quad T<T_{X}
$$

where we will assume that $\widetilde{\Delta}_{c l}(T)$ has the same temperature dependence as the BCS superconducting gap function and vanishes at the mean field critical temperature $T_{c}^{M F}$. More specifically, as it will be clear later from the expression for the self-energy in the pseudogap regime, $\widetilde{\Delta}_{c l}^{2}(T)=\Delta_{\mathbf{k}}^{2}(T) / g_{\mathbf{k}}^{2}$. We also note that having the crossover temperature to the pseudogap regime of the order of $T_{c}^{M F}$ is consistent with recent data [17 which show $T^{*} \sim T_{c}^{M F}$. Since the largest contribution in the frequency integral comes from $\omega \ll T$ in the critical regime $\left(T<T_{X}\right)$, eq. (4) can be approximated by:

$$
T \int_{0}^{\bar{q}_{c}} \frac{d^{3} q}{\Omega} \chi(0, \mathbf{q})=\widetilde{\Delta}_{c l}^{2}(T)
$$

Here $\bar{q}_{c}$ is a momentum space cutoff. In the in plane direction, we set $\bar{q}_{c}^{(x y)}=q_{c}=\pi / \xi_{0}$. In the out of plane direction, the quantity $\pi / \xi_{0 z}=\pi / \gamma \xi_{0}$ can become larger than the Brillouin zone boundary $q_{B Z}^{(z)}$ for sufficiently small $\gamma$, and hence we chose $\bar{q}_{c}^{(z)}=q_{c}^{(z)}$ to be

$$
q_{c}^{(z)}=\min \left[q_{c} / \gamma, q_{B Z}^{(z)}\right]
$$

We checked that the exact value of the cutoff does not significantly affect the results.

With the help of equations (2) and (5), we can obtain the temperature dependence of the correlation length $\xi[18$ :

$$
\frac{q_{c}^{(z)}}{q_{B Z}^{(z)}}\left\{\left(1+\left(q_{c} \xi\right)^{-2}\right)\left(\frac{\sqrt{1+\left(q_{c} \xi\right)^{-2}}}{\sqrt{1-\widetilde{\gamma}^{2}}} \operatorname{atanh} \frac{\sqrt{1-\widetilde{\gamma}^{2}}}{\sqrt{1+\left(q_{c} \xi\right)^{-2}}}-\frac{\operatorname{atan}\left(\widetilde{\gamma} q_{c} \xi\right)}{\widetilde{\gamma} q_{c} \xi}\right)-\frac{1}{3}\right\}=\frac{T_{0}}{T} \frac{\widetilde{\Delta}_{c l}^{2}(T)}{\widetilde{\Delta}_{c l}^{2}(0)}
$$


where $\widetilde{\gamma}=\gamma q_{c}^{(z)} / q_{c}$. The parameter $T_{0}$ is defined by:

$$
T_{0}=2 \pi \xi_{0}^{2} \widetilde{\Delta}_{c l}^{2}(0) / \chi_{0}
$$

The meaning of $T_{0}$ is particularly clear in two dimensions, in which from eq. (7) $\xi \propto e^{\frac{T_{0}}{T}}$. In the weak coupling limit, $\xi_{0} \sim \frac{v_{F}}{\Delta}$ and $T_{0}$ is proportional to the Fermi energy and therefore is very large compared to $T_{c}^{M F}$. As we shall see below, this implies an extremely narrow fluctuation regime unless $\gamma$ is practically zero. On the other hand, in the intermediate coupling regime we can expect $\xi_{0} \sim 1$, which leads to a sufficiently large fluctuation regime. In the rest of this work we will choose $\xi_{0} \sim 1$, and $T_{0} \sim \Delta_{\max }(0)$ unless otherwise stated (in particular all Figures have being generated by choosing $\xi_{0}=2.0$, and $\left.T_{0}=\Delta_{\max }(0)\right)$.

From equation (7) we can find the superconducting transition temperature, by letting $\xi \rightarrow \infty:$

$$
T_{c}(\gamma)=\frac{q_{B Z}^{(z)}}{q_{c}^{(z)}}\left[\frac{\operatorname{atanh}\left(\sqrt{1-\widetilde{\gamma}^{2}}\right)}{\sqrt{1-\widetilde{\gamma}^{2}}}-\frac{1}{3}\right]^{-1} \frac{\Delta^{2}\left(T_{c}\right)}{\Delta^{2}(0)} T_{0}
$$

This is still an implicit equation for $T_{c}$, but it clearly shows two important points. First, $T_{c}(\gamma)$ is a monotonically increasing function of $\gamma$, and as the interplane anisotropy increases $(\gamma \rightarrow 0)$, the critical temperature approaches zero logarithmically in $\gamma$, therefore satisfying the Mermin-Wagner theorem. Clearly in this limit, a Kosterlitz-Thouless (KT) transition may set in at some finite temperature, but we will not consider this possibility here and we will assume that the SC critical temperature due to $3 \mathrm{D}$ effects is higher then $T_{c}^{K T}$. Second, for any finite value of the anisotropy parameter $\gamma, T_{c}$ is a monotonically increasing function of $T_{0}$, saturating at $T_{c}^{M F}$ for $T_{0} \gg T_{c}^{M F}$. That is, in the weak coupling limit, the temperature range of the pseudogap phase is very small even for quasi $2 \mathrm{D}$ materials, while for intermediate coupling, the temperature range of the pseudogap phase can be appreciable.

We are now in a position to estimate the imaginary part of the self energy. Close to the SC transition temperature, classical fluctuations give a dominant contribution to the self energy. We can therefore approximate eq. (1) with its classical part $\Sigma \approx \Sigma_{c l}$. Using (11), (2), and [18 the imaginary part of the self energy $\Sigma_{c l}^{\prime \prime}$ can be evaluated analytically: 


$$
\begin{aligned}
\Sigma^{\prime \prime}(\omega, \mathbf{k}, \gamma, T)= & -\frac{\pi}{2} \frac{q_{c}^{(z)}}{q_{B Z}^{(z)}} \frac{\Delta_{\mathbf{k}}^{2}(0)}{v_{\mathbf{k}} q_{c}} \frac{T}{T_{0}} \theta\left(v_{\mathbf{k}} q_{c}-|\omega|\right)\left[-\frac{1}{2}\left(1-\left(\frac{\omega}{v_{\mathbf{k}} q_{c}}\right)^{2}\right)+\left(1+\left(q_{c} \xi\right)^{-2}\right) \times\right. \\
& \left.\frac{1}{\widetilde{\gamma}} \ln \frac{\widetilde{\gamma} \sqrt{1+\left(q_{c} \xi\right)^{-2}}+\sqrt{\widetilde{\gamma}^{2}+\left(q_{c} \xi\right)^{-2}+\left(1-\widetilde{\gamma}^{2}\right)\left(\frac{\omega}{v_{\mathbf{k}} q_{c}}\right)^{2}}}{(1+\widetilde{\gamma}) \sqrt{\left(q_{c} \xi\right)^{-2}+\left(\frac{\omega}{v_{\mathbf{k}} q_{c}}\right)^{2}}}\right]
\end{aligned}
$$

In this expression we have taken into consideration only the most important contribution coming from the pole in $\chi$ at $i \omega=D\left(\xi^{-2}+q_{x}^{2}+q_{y}^{2}+\gamma^{2} q_{z}^{2}\right) \ll T$. Moreover, we expanded the single particle energy up to first order in $\mathbf{q}\left(\epsilon(\mathbf{q}-\mathbf{k}) \simeq \epsilon(-\mathbf{k})+\mathbf{v}_{-\mathbf{k}} \cdot \mathbf{q}\right.$, where $\mathbf{k}$ is

on the Fermi surface and we use inversion symmetry to set $\mathbf{v}_{-\mathbf{k}}=-\mathbf{v}_{\mathbf{k}}$ ), and integrated in momentum space using the cutoff $\bar{q}_{c}$. Notice that this momentum integration is essential in order to account for the effect of dimensionality. Indeed, neglecting the $\mathbf{q}$ dependence in the Green's function in eq. (1) would lead to a pseudogap in all dimensions. The factor $\theta\left(v_{\mathbf{k}} q_{c}-|\omega|\right)$ also comes from this momentum integration and introduces a convenient frequency cutoff. The real part of the self energy $\Sigma^{\prime}$ can be obtained numerically for arbitrary $\gamma$ by using the Kramers-Kronig relations. Notice that in the strictly two dimensional case $(\gamma=0)$, the real and imaginary part of the self energy can both be expressed analytically [9].

\section{III. $\mathbf{T}=\mathbf{T}_{C}(\gamma)$ :}

We will start our study of the effect of dimensionality by analyzing the spectral function when the pseudogap effect is the most noticeable, that is at $T=T_{c}(\gamma)$ where the correlation length $\xi=\infty$. At exactly the phase transition the self-energy is singular, and this leads to a pseudogap at $T=T_{c}$. However, as we will see below, the qualitative shape of the spectral function and the strength of the pseudogap differ dramatically in 2D and 3D.

In Fig.(国) the spectral function $A\left(\omega, \mathbf{k}, \gamma, T=T_{c}(\gamma)\right)$ is plotted for three distinct values of the anisotropy parameter $(\gamma=0,0.1,1)$, with each curve plotted at the respective SC transition temperature $T_{c}(\gamma)$, all other parameters being the same. The spectral function in $2 \mathrm{D}, A\left(\omega, \mathbf{k}, 0, T=T_{c}(0)=0\right)$, is simply given by two sharp peaks located at $\omega= \pm \Delta_{\mathbf{k}}(0)$ 
[19]. On the other hand, in the isotropic 3D case $(\gamma=1)$, the spectral function consists of two broad maxima very close to each other; although $A\left(\omega, \mathbf{k}, \gamma=1, T=T_{c}(1)\right)$ vanishes right at $\omega=0$ because of the singularity in $\Sigma^{\prime \prime}$, it is small only in an extremely narrow range of frequencies. As suggested by the $\gamma=0.1$ curve, a fairly large anisotropy is required to obtain two well separated peaks.

In order to fully understand the effect of dimensionality, we have to take a closer look at the analytic properties of the self energy at small energies, for different values of the anisotropy $\gamma$, at $T=T_{c}(\gamma)$. The imaginary and the real part of the self-energy are plotted in Fig. (2.a) and (2.b) respectively for the same three values of $\gamma$ used in Fig. (11). In exactly two dimensions, in which case $T_{c} \rightarrow 0$, the imaginary part of the self energy is a delta function, and the real part of the self-energy is given by $\Delta_{\mathbf{k}}^{2}(0) / \omega$. On the other hand, in case of an isotropic three dimensional superconductor, at $T=T_{c}(\gamma=1)$, the imaginary part of the self energy only diverges logarithmically at $\omega=0$, while the real part is discontinuous $\left(\Sigma^{\prime}(\omega) \propto \operatorname{sign}(\omega)\right)$ but finite and small at $\omega=0$.

For large, but finite anisotropy $(\gamma \ll 1)$, a crossover frequency $\omega_{X}=\gamma v_{\mathbf{k}} q_{B Z}^{(z)}$ appears in the frequency dependence of $\Sigma(\omega)$. For $\omega<\omega_{X}$ the behavior of the self energy is similar to the isotropic 3D behavior, while for $\omega>\omega_{X}$ it has essentially the 2D character. More specifically, for $\omega \ll \omega_{X}$ the imaginary part of the self energy $\Sigma^{\prime \prime}\left(\omega, \mathbf{k}, \gamma, T=T_{c}(\gamma)\right)$ diverges logarithmically at $\omega=0$, while a Taylor series expansion of the real part of the self energy yields:

$$
\Sigma^{\prime}\left(\omega, \mathbf{k}, \gamma, T=T_{c}(\gamma)\right) \simeq \frac{\Delta_{\mathbf{k}}^{2}(0)}{\omega_{X}} \frac{T_{c}(\gamma)}{T_{0}}\left\{\frac{\pi^{2}}{4} \operatorname{sign}(\omega)-\left(1+\widetilde{\gamma}^{2}\right) \frac{q_{B Z}^{(z)}}{q_{c}^{(z)}} \frac{\omega}{\omega_{X}}\right\}
$$

which consists of a finite discontinuity at $\omega=0$, to be contrasted with the infinite discontinuity in $2 \mathrm{D}$.

This behavior is similar to that of the isotropic three dimensional superconductor. As the anisotropy is increased $(\gamma \rightarrow 0)$ the jump in $\Sigma^{\prime}$ at $\omega=0$ and the magnitude of the slope in eq. (10) gradually increase to resemble the divergence of $\Sigma^{\prime}(\omega \rightarrow 0)$ in the pure 2D case (see Fig. (2.b)). Now consider excitation energies $\omega \gg \omega_{X}$ and $\gamma \ll 1$. In this case 
the imaginary part of the self energy $\Sigma^{\prime \prime}\left(\omega, \mathbf{k}, \gamma, T=T_{c}(\gamma)\right)$ crosses over to a $\sim 1 / \omega$ power law behavior, and the real part of the self energy is well approximated by the following asymptotic expansion:

$$
\Sigma^{\prime}\left(\omega, \mathbf{k}, \gamma, T=T_{c}(\gamma)\right) \simeq \frac{\Delta_{\mathbf{k}}^{2}\left(T_{c}(\gamma)\right)}{\omega}\left(1+\frac{\frac{4}{3}+\log \left(\frac{\omega}{v_{\mathbf{k}} q_{c}}\right)-\frac{3}{2}\left(\frac{\omega}{v_{\mathbf{k}} q_{c}}\right)^{2}}{\log \left(\frac{2}{\gamma}\right)-\frac{1}{3}}\right)
$$

This is the $\Delta^{2} / \omega$ dependence expected for the two dimensional case just before the SC phase transition $T \rightarrow T_{c}=0$, plus corrections which vanish logarithmically in $\gamma$ as $\gamma \rightarrow 0$. The two dimensionality of this behavior is made absolutely clear by the observation that eq. (11) coincides with the asymptotic expansion of the real part of the self energy calculated in two dimensions at $T=T_{c}(\gamma)$. That is for $\omega \gg \omega_{X}$, the third dimension is no longer relevant. In the next section we shall see that this collapse of the self energy to its two dimensional limit persists and actually extends to lower excitation energies as the temperature is increased. Fig. (3) exemplifies the asymptotics just discussed for the real part of the self energy.

The role of the crossover energy $\omega_{X}=\gamma v_{\mathbf{k}} q_{B Z}^{(z)}$ in the experimental observation of the pseudogap, such as in ARPES data, can be fully appreciated if $\omega_{X}$ is compared to the gap energy $\Delta_{\mathbf{k}}(0)$. If $\Delta_{\mathbf{k}}(0) \ll \omega_{X}(\mathbf{k})$, the properties of the pseudogap will be 3D like. The position of the maxima in the spectral function can be estimated by solving $\omega-\Sigma^{\prime}(\omega)=0$, where in this limit eq. (10) can be used for $\Sigma^{\prime}(\omega)$. The half width of the pseudogap, $\Delta_{\mathrm{pg}}\left(\mathbf{k}, \gamma, T=T_{c}(\gamma)\right)$, at the transition temperature is roughly proportional to:

$$
\frac{\Delta_{\mathrm{pg}}\left(\mathbf{k}, \gamma, T=T_{c}(\gamma)\right)}{\Delta_{\mathbf{k}}(0)} \propto \frac{\pi^{2}}{4} \frac{T_{c}(\gamma)}{T_{0}} \frac{\Delta_{\mathbf{k}}(0)}{\omega_{X}(\mathbf{k})}
$$

Therefore in case of a mildly anisotropic material, the pseudogap in the spectral function may be completely unobservable, especially in the weak coupling limit where $T_{0} \sim E_{F}$.

The situation changes considerably if high anisotropies are attained $(\gamma \ll 1)$, and $\Delta_{\mathbf{k}}(0) \gg \omega_{X}(\mathbf{k})$. In this limit the spectral function is very well described by its two dimensional limit except in a narrow range of excitation energies $|\omega| \lesssim \omega_{X}(\gamma)$, and the width of the pseudogap is expected to be of the order of $2 \Delta_{\mathbf{k}}(0)$. 
Notice that the relation between $\Delta_{\mathbf{k}}(0)$ and $\omega_{X}=\gamma v_{\mathbf{k}} q_{B Z}^{(z)}$ is $\mathbf{k}$ dependent, that is it depends upon the particular point probed in the Brillouin zone. In the case of high temperature superconductors, points on the Fermi surface close to the zone diagonal have small values of $\Delta_{\mathbf{k}}(0)$, and relatively high values of the Fermi velocity. On the basis of this analysis for these points, the pseudogap effect should not be observable, or should be very small even at $T=T_{c}$. On the other hand, points on the Fermi surface close to the $(\pi, 0)$ point have large values of the gap, and small values of the Fermi velocity, and here the pseudogap effect should be the strongest, as indeed is experimentally observed [3].

\section{IV. $\mathbf{T} \geq \mathbf{T}_{C}(\gamma)$}

Let's now consider how the pseudogap evolves with temperature. For a given $\gamma$, as soon as the temperature is larger than $T_{c}(\gamma)$, the singularities in the real and imaginary part of the self energy at $\omega=0$ disappear. The singularity in $\Sigma^{\prime \prime}(\omega)$ is transformed into a peak of finite height, which decreases and widens as the temperature increases, so that the spectral function $A(\omega, \mathbf{k})$ does not vanish anymore at $\omega=0$, and the pseudogap begins to fill in. The discontinuity in $\Sigma^{\prime}(\omega)$ is replaced by a line with a finite slope given by:

$$
\left.\frac{\partial \Sigma^{\prime}(\omega, \mathbf{k}, \gamma, T)}{\partial \omega}\right|_{\omega=0}=\frac{\Delta_{\mathbf{k}}^{2}(0)}{\left(v_{\mathbf{k}} q_{c}\right)^{2}} \frac{T}{T_{0}} \frac{q_{c}^{(z)}}{q_{B Z}^{(z)}}\left[\frac{1+\left(q_{c} \xi\right)^{-2}}{\widetilde{\gamma}}\left(q_{c} \xi\right) \operatorname{atan}\left(\widetilde{\gamma} q_{c} \xi\right)-1\right]
$$

As is clear from this equation, this slope is infinite at $T=T_{c}(\gamma)$ (since $\xi \rightarrow \infty$ ), and decreases with increasing temperature (since $\xi$ decreases). As long as this slope is larger than one, the equation $\omega-\Sigma^{\prime}(\omega, \mathbf{k}, \gamma, T)=0$ has two non-zero solutions, and the spectral function has two distinct peaks and a minimum at $\omega=0$. When $\left(\partial \Sigma^{\prime} / \partial \omega\right)_{\omega=0}<1$, the equation $\omega-\Sigma^{\prime}(\omega, \mathbf{k}, \gamma, T)=0$ has only the solution $\omega=0$. Due to the maximum in $\Sigma^{\prime \prime}(\omega, \mathbf{k}, \gamma, T)$ at $\omega=0$, this may still correspond to a minimum in $A(\omega=0, \mathbf{k})$, but the pseudogap is quickly suppressed as $\left(\partial \Sigma^{\prime} / \partial \omega\right)_{\omega=0}$ is further reduced from 1. Figures (5.a) and (5.b) are examples of the temperature evolution of the spectral function provided by this model. 
The dimensional crossover energy, and its relation with the gap amplitude $\Delta_{\mathbf{k}}(0)$, still determine the behavior of the self energy and hence of the spectral function of the material. We have already noticed in the previous section that, for $\gamma \ll 1$ and $\omega \gg \omega_{X}$, the self energy of the anisotropic superconductor at the transition temperature $T_{c}(\gamma)$ coincides with that of the two-dimensional one, and therefore if $\Delta_{\mathbf{k}}(0) \gg \omega_{X}(\mathbf{k})$ the values of the spectral function in these two cases coincide except in a narrow range of excitation energies $\omega \lesssim \omega_{X}$. This behavior persists as the temperature is increased above $T_{c}(\gamma)$. This is because the correlation length in the third dimension, $\xi_{z}$, decreases much faster (by a factor of $1 / \gamma$ ) than the in plane correlation length $\xi$, and eventually becomes smaller than the interplane separation $a_{z}$, thus reducing the material to a stack of nearly independent two dimensional planes. Therefore, at temperatures high enough that $\xi_{z} \ll a_{z}$, while still $\xi \gg a$, the relation:

$$
\Sigma(\omega, \mathbf{k}, \gamma, T) \simeq \Sigma_{2 D}(\omega, \mathbf{k}, T)
$$

is valid for all excitation energies and not only for $\omega \gg \omega_{X}$ as we found at $T=T_{c}(\gamma)$. Therefore if $\Delta_{\mathbf{k}}(0) \gg \omega_{X}(\mathbf{k})$, the temperature evolution of the pseudogap in the spectral function of an anisotropic material is entirely two dimensional.

For $\Delta_{\mathbf{k}}(0)<\omega_{X}(\mathbf{k})$, there is no such general relation as eq. (14). The pseudogap is characterized by a small separation of the broad maxima in the spectral function, at least for small to intermediate coupling, and a small temperature range for which these maxima are distinct.

In order to estimate the temperature $T^{*}$ at which the pseudogap closes, we can use the condition for having non-zero solutions of $\omega-\Sigma^{\prime}(\omega, \mathbf{k}, \gamma, T)=0$, which ceases to be valid at a temperature $T^{*}$ such that:

$$
\left.\frac{\partial \Sigma^{\prime}\left(\omega, \mathbf{k}, \gamma, T^{*}\right)}{\partial \omega}\right|_{\omega=0} \approx 1
$$

Figure (4) is a plot of $T^{*} / T_{M F}$ as a function of the ratio $\Delta_{\mathbf{k}}(0) / v_{\mathbf{k}}$. Clearly in the limit $\Delta_{\mathbf{k}}(0) / v_{\mathbf{k}} \rightarrow 0, T^{*} \rightarrow T_{c}(\gamma)$, which means that close to a node the pseudogap in the spectral function can open only in an extremely small temperature range. As the ratio $\Delta_{\mathbf{k}}(0) / v_{\mathbf{k}}$ 
increases, $T^{*} / T_{c}^{M F}$ increases for any value of $\gamma$, but the range of the pseudogap effect in the spectral function $\left(T^{*}-T_{c}(\gamma)\right)$ increases more rapidly for higher anisotropies, so that it is larger the larger the anisotropy. Notice also that the curves at high anisotropy (small $\gamma$ ) collapse on top of the $\gamma=0$ curve as soon as $\Delta_{\mathbf{k}}(0) \gtrsim \omega_{X}(\gamma, \mathbf{k})$. This is a consequence of the dimensional crossover just discussed (eq. (14)): the pseudogap in the spectral function will disappear at the same temperature for anisotropic materials with different $\gamma$ as long as $\Delta_{\mathbf{k}}(0) \gg \omega_{X}(\gamma, \mathbf{k})$

It is important to notice once again that the condition $\Delta_{\mathbf{k}}(0) \gg \omega_{X}(\gamma, \mathbf{k})$ is $\mathbf{k}$ dependent. This is in qualitative agreement with the experimentally observed phenomenon of the Fermi arcs [3]. It is apparent from photoemission experiments that $T^{*}$ depends on which points of the Fermi surface are probed. The general trend is that the pseudogap near the $(0,0)-(\pi, \pi)$ direction closes at a lower temperature than that near the $(\pi, 0)$ one. This is well captured by eq. (15) and (13). For points on the Fermi surface close to the zone diagonal, the Fermi velocity $v_{\mathbf{k}}$ is at its maximum, while at the same time, if a d-wave symmetry for the order parameter is assumed, $\Delta_{\mathbf{k}}(0)$ is small. This makes the ratio $\Delta_{\mathbf{k}}(0) / v_{\mathbf{k}}$ small and therefore $T^{*}$ small (see Fig. (团)). Conversely, close to $(\pi, 0)$ the Fermi velocity $v_{\mathbf{k}}$ is at its minimum, and for a d-wave order parameter, $\Delta_{\mathbf{k}}(0)$ is large, which makes $T^{*}$ large.

Finally we want to comment on another peculiarity of the temperature evolution of the pseudogap which is in good qualitative agreement with the experimentally observed phenomenon of the closing up versus filling in behavior of the pseudogap at different points in k-space. Fig. (5.a) and (5.b) exemplify the contrast in the temperature evolution of the spectral function for larger and smaller values of the $\mathbf{k}$ dependent ratio $\Delta_{\mathbf{k}}(0) / v_{\mathbf{k}}$, which resembles quite closely the ARPES experimental data. For points on the Fermi surface close to $(\pi, 0)$, the ratio $\Delta_{\mathbf{k}}(0) / v_{\mathbf{k}}$ is relatively large, and the spectral function evolves like in Fig. (5.a), that is the peaks seem rather immobile until the temperature is close to $T_{c}^{M F}$ and the pseudogap appears to fill up. On the other hand, for points close to the zone diagonal, the ratio $\Delta_{\mathbf{k}}(0) / v_{\mathbf{k}}$ tends to be small and the behavior is more like that of Fig. (5.b), that is 
the pseudogap appears to close in rapidly with temperature. However in Fig. (5.a) another interesting feature is evident: the separation of the peaks as the temperature is increased increases a little at first before it starts to collapse. It can be shown that this effect is a consequence of our assumption that $\Delta_{\mathbf{k}}(T)$ is given by the BCS expression, which is almost constant at temperatures $T \ll T_{c}^{M F}$. A relaxation of this assumption could eliminate the effect. Further investigation of this effect would require a more accurate model for $\Delta_{\mathbf{k}}(T)$, which is beyond the scope of this paper.

\section{CONCLUSIONS}

We have demonstrated that classical pairing fluctuations give rise to a pseudogap phenomenon in the limit of small to intermediate coupling strength. However, in both 2D and 3D, the dimensionality plays a crucial role in the strength, shape, and temperature dependence of the pseudogap. In three dimensional isotropic or weakly anisotropic systems, the effect is very weak. The magnitude of the pseudogap just above the transition temperature is reduced from its $T=0$ value at least by a factor of the order of $\Delta_{\mathbf{k}}(0) / \gamma v_{\mathbf{k}} q_{B Z}^{(z)}$, which in the case of an isotropic superconductor would be of the order of $\Delta / E_{F}$, and its temperature range is quite small. Large values of the coupling strength are therefore necessary to observe a pseudogap in a material with small anisotropy. On the other hand, in two dimensions, or for relatively high anisotropy, the pseudogap is a sizeable effect even for small to intermediate coupling strength: the magnitude of the pseudogap just above the transition temperature is almost the same as its $T=0$ value, while its temperature range is also considerably increased, $T^{*}$ being of the order of $T_{c}^{M F}$ provided that $\xi_{0} \sim 1$. The dimensional crossover between these two qualitatively different regimes is defined by $\gamma \sim \Delta_{\mathbf{k}}(0) / v_{\mathbf{k}} q_{B Z}^{(z)}$. For val-

ues of $\gamma$ significantly smaller than $\Delta_{\mathbf{k}}(0) / v_{\mathbf{k}} q_{B Z}^{(z)}$, the pseudogap is describable in terms of the two dimensional limit, while for $\gamma$ essentially larger than $\Delta_{\mathbf{k}}(0) / v_{\mathbf{k}} q_{B Z}^{(z)}$, the anisotropic three dimensional limit is proper. Furthermore, the in plane anisotropy plays a crucial role in determining the temperature range of the pseudogap through the ratio $\Delta_{\mathbf{k}}(0) / v_{\mathbf{k}}$, so that 
close to the $(\pi, 0)$ point, the Fermi velocity is small (Van Hove point) and $T^{*}$ is large, while for points on the Fermi surface for which the ratio $\Delta_{\mathbf{k}}(0) / v_{\mathbf{k}}$ is small, the pseudogap has a smaller range.

The parameters characterizing the underdoped cuprates, namely high anisotropies, large superconducting gap amplitudes, and small correlation lengths, put these materials in the 2D limit, and our results are in rather good qualitative agreement with experiment.

\section{ACKNOWLEDGMENTS}

This work was supported by the U. S. Dept. of Energy, Basic Energy Sciences, under contract W-31-109-ENG-38, and the National Science Foundation DMR 91-20000 through the Science and Technology Center for Superconductivity.

[1] For a recent review see M. Randeria, Proceedings of the International School of Physics, "Enrico Fermi", Varenna, 1997 (North Holland, New, York, in press), cond-matt/9710223.

[2] D.S. Marshall, D.S. Dessau, A.G. Loeser, C-H.Park, A.Y.Matsuura, J. N. Eckstein, I. Bozovic, P. Fournier, A. Kapitulnik, W.E. Spicer, and Z.-X. Shen, Phys. Rev. Lett. 76, 4841 (1996); H. Ding, T. Yokoya, J.C. Campuzano, T. Takahashi, M. Randeria, M. R. Norman, T. Mochiku, K. Kadowaki, and J. Giapintzakis, Nature 382, 51 (1996); A.G. Loeser, Z.-X. Shen, D.S. Dessau, D.S. Marshall, C.H. Park, P. Fournier, A. Kapitulnik, Science 273, 325 (1996).

[3] M.R. Norman, H Ding, M. Randeria, J.C. Campuzano, T. Yokoya, T Takeuchi, T. Takahashi, T. Mochiku, K. Kadowaki, G. Guptasarma, and D.G. Hinks, Nature 392, 157 (1998).

[4] J.U Lee, P. Guptasarma, D. Hombaker, A. El-Kortas, D. Hinks, and K.E. Gray, Appl. Phys. Lett. 71, 10 (1997).

[5] P.A. Lee, N. Nagaosa, T.-K. Ng, and X.-G. Wen, Phys. Rev. B 57, 6003 (1998); P.W. Anderson, 
The Theory of Superconductivity in High-Tc Cuprate Superconductors (Princeton University Press, Princeton 1997).

[6] A.V. Chubukov, D. Pines, B. Stojković, J. Phys. Cond. Matt. 8, 10017 (1996).

[7] M. Randeria, N. Trivedi, A. Moreo, and R. T. Scalettar, Phys. Rev. Lett. 69, 2001 (1992); R. Micnas, M.H. Pedersen, S. Schaforth, and T. Schneider, J. J. Rodríguez-Núñez and H. Beck, Phys. Rev. B 52, 16223 (1995).

[8] Q. Chen, Ioan Kosztin, Boldizár Jankó, and K. Levin, cond-matt/9805032.

[9] Y. M. Vilk and A.-M. S. Tremblay, Europhys. Lett. 33, 159 (1996); Y. M. Vilk and A.-M. S. Tremblay, J. Phys. I France 7, 1309 (1997).

[10] Y. M. Vilk, Phys. Rev. B 55, 3870 (1997).

[11] Although this expression is strictly speaking valid only in the weak coupling limit, it can actually be extended up to intermediate coupling by replacing the bare interaction with a renormalized one. See for example [9].

[12] J. Maly, B. Janko, and K. Levin, cond-matt/9710187.

[13] J. C. Campuzano, G. Jennings, M. Faiz, L. Beaulaigue, B.W. Veal, J.Z. Liu, A.P. Paulikas, K. VanderVoort, and H. Claus, Phys. Rev. Lett. 64, 2308 (1990); C. G. Olson, R. Liu, D.W. Lynch, R.S. List, A.J. Arko, B.W. Veal, Y.C. Chang, P.Z. Jiang, and A.P. Paulikas, Phys. Rev. B 42, 381 (1990).

[14] N.D. Mermin and H. Wagner, Phys. Rev. Lett. 17, 1133 (1966).

[15] The factor $\eta_{i j}$ determines the symmetry of the order parameter. For an s-wave superconductor $\eta_{i j}=\delta_{i j}$, while for a d-wave superconductor $\eta_{i j}=\delta_{i, j+\widehat{x}}+\delta_{i, j-\widehat{x}}-\delta_{i, j+\widehat{y}}-\delta_{i, j-\widehat{y}}$.

[16] J.M. Singer, M.H. Pedersen, T. Schneider, H. Beck, H.-G. Mattutis, Phys. Rev. B 54, 1286 (1996). 
[17] M. Oda, K. Hoya, R. Kubota, C. Manabe, N. Monomo, T. Nakano, M. Ido, Physica C 281, 135 (1997); H Ding, J.C. Campuzano, M.R. Norman, M. Randeria, T. Yokoya, T. Takahashi, T Takeuchi, T. Mochiku, K. Kadowaki, G. Guptasarma, and D.G. Hinks, to be published, J. Phys. Chem. Solids.

[18] In obtaining this equation we have used a "soft" cutoff procedure, which ensures that $\chi(0, \mathbf{q})$ approaches 0 continuously at $q=q_{c}$, in order to avoid the spurious singularities that a sharp cutoff would give rise to in the self energy. For this particular calculation, we have replaced $\chi_{0}$ with $\chi_{0} U(\mathbf{q})$ with $U(\mathbf{q})=1-q_{c}^{-2}\left(q_{x}^{2}+q_{x}^{2}+\gamma^{2} q_{x}^{2}\right)$.

[19] In this and other plots of the spectral function, a broadening factor $\Gamma_{1}=0.1 \times \Delta_{\mathbf{k}}(0)$ has being added to the imaginary part of the self energy, in order to make the sharp peaks visible.

[20] A.-M. Daré, Y. M. Vilk, and A.-M. S. Tremblay, Phys. Rev. B 53, 14236 (1996).

[21] M.R. Norman, M. Randeria, H. Ding, and J. C. Campuzano, Phys. Rev. B 57, R11093 (1998). 


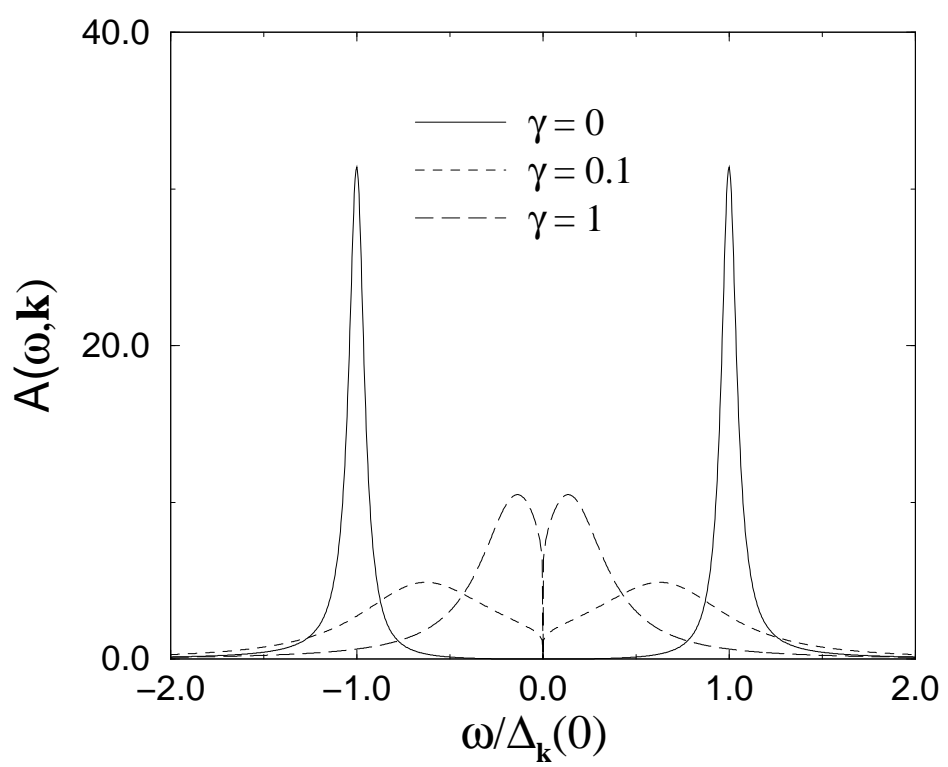

FIG. 1. Plot of the spectral function [19] $A\left(\omega, \mathbf{k}, \gamma, T=T_{c}(\gamma)\right)$ as a function of $\omega$ for three different values of the anisotropy parameter $\gamma$, and the same value of the ratio $\Delta(\mathbf{k}) / \pi v_{\mathbf{k}}=0.064$. Each spectral function is plotted at the transition temperature corresponding to $\gamma$. 

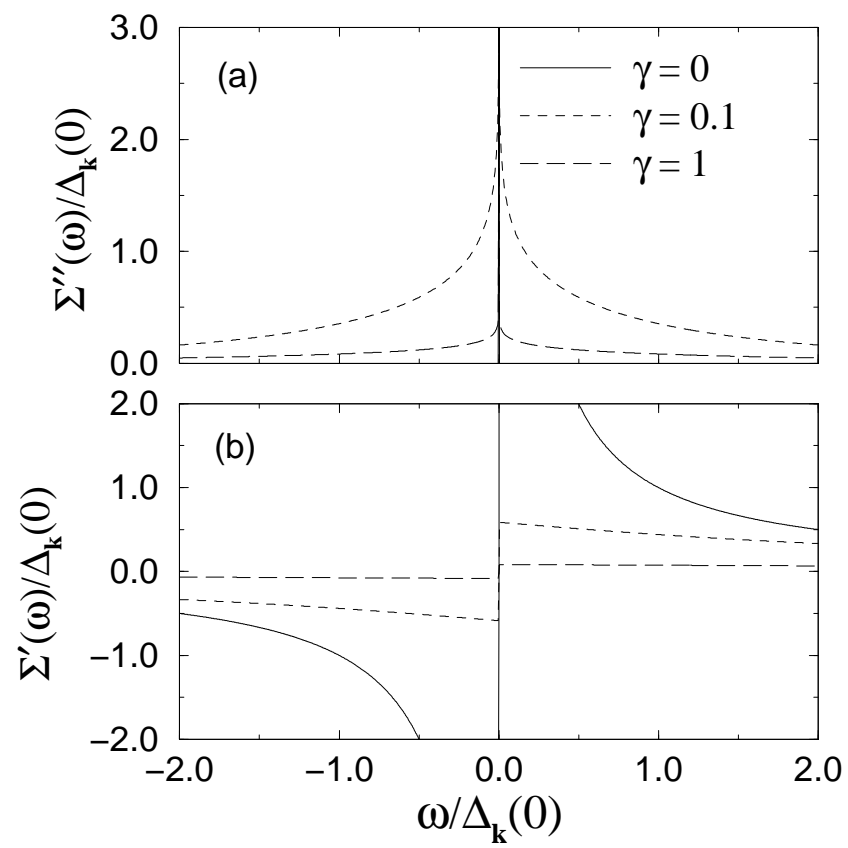

FIG. 2. Plot of (a) the imaginary part of the self-energy $\Sigma^{\prime \prime}\left(\omega, \mathbf{k}, \gamma, T=T_{c}(\gamma)\right)$ and (b) the real part of the self-energy $\Sigma^{\prime}\left(\omega, \mathbf{k}, \gamma, T=T_{c}(\gamma)\right)$ as functions of $\omega$, of three different values of the anisotropy parameter $\gamma$, and the same value of the ratio $\Delta(\mathbf{k}) / \pi v_{\mathbf{k}}=0.064$. Each self-energy is plotted at the transition temperature corresponding to $\gamma$. 


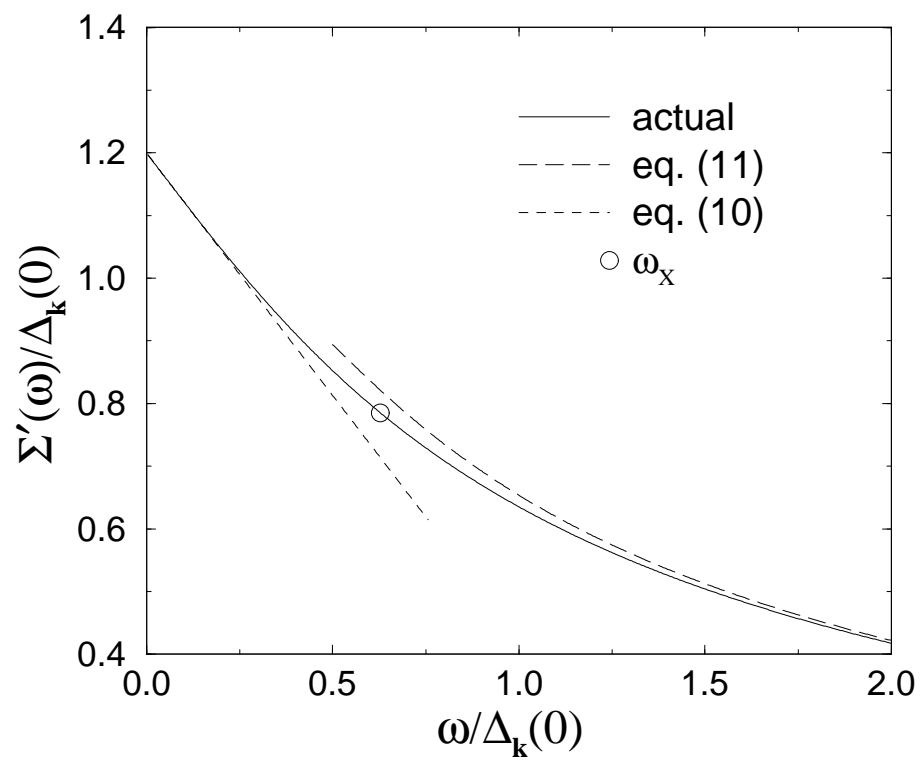

FIG. 3. Plot of the real part of the self energy $\Sigma^{\prime}(\omega, \mathbf{k})$ solid line, as a function of $\omega$, compared with the asymptotic expansions given in eq. (11), long dashed line, and eq. (10), dashed line. The value of $\Sigma^{\prime}(\omega)$ at the dimensional crossover frequency $\omega_{X}=\gamma v_{\mathbf{k}} q_{B Z}^{(z)}$, is indicated by a circle. All curves are plotted for the same value of the anisotropy parameter $\gamma=0.04$.

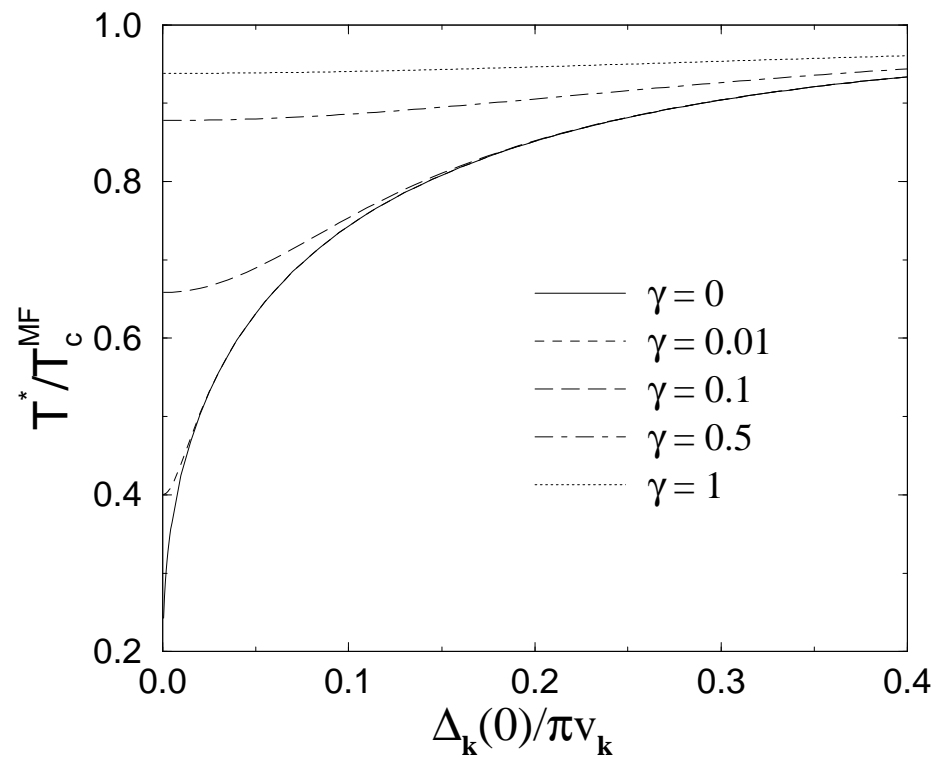

FIG. 4. Plot of the estimated temperature $T^{*}$ for the closing of the pseudogap, as a function of the ratio $\Delta(\mathbf{k}) / \pi v_{\mathbf{k}}$ for various values of the anisotropy parameter $\gamma$. 


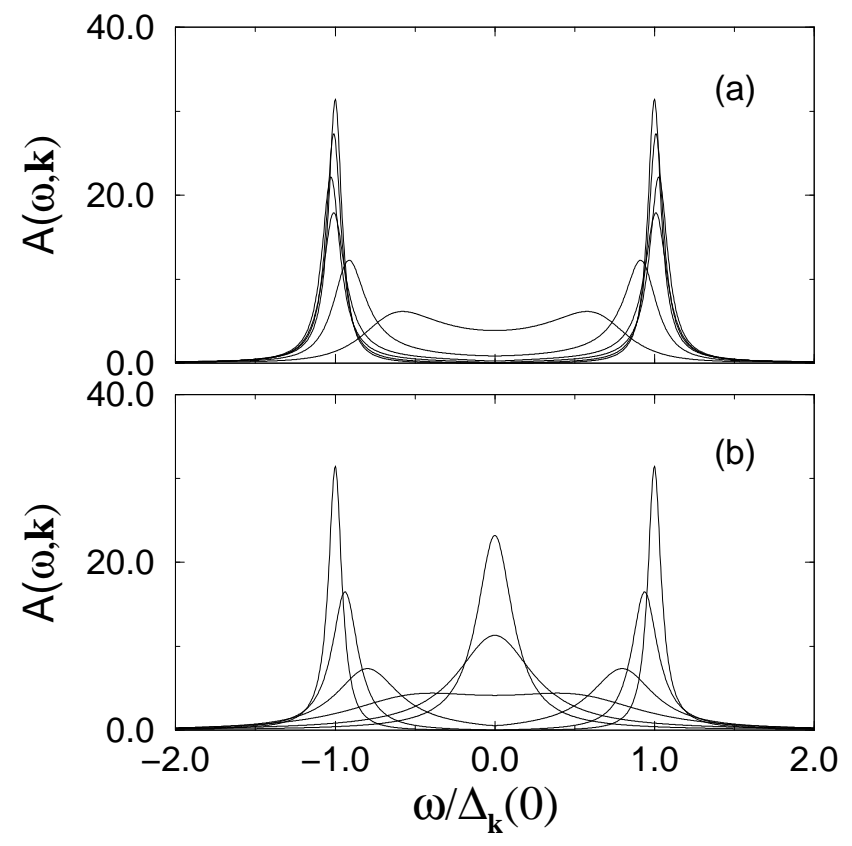

FIG. 5. Plot of the spectral function [19] $A(\omega, \mathbf{k}, \gamma=0)$ in $2 \mathrm{D}$ as a function of $\omega$, at various temperatures $\left(T / T_{c}^{M F}=0.0,0.1,0.3,0.5,0.7,0.9\right)$ for two different values of the ratio $\Delta(\mathbf{k}) / v_{\mathbf{k}}$ : (a) $\Delta(\mathbf{k}) / \pi v_{\mathbf{k}}=0.32$ and (b) $\Delta(\mathbf{k}) / \pi v_{\mathbf{k}}=0.016$. 